\title{
A CMOS-based ISFET Chemical Imager with Auto-Calibration Capability
}

\author{
Clinton Z. D. Goh, Pantelis Georgiou, Member, IEEE, Timothy G. Constandinou, Senior Member, IEEE, \\ Themistoklis Prodromakis, Member, IEEE, and Christofer Toumazou Fellow, IEEE
}

\begin{abstract}
This paper presents a novel auto-calibration technique for eliminating sensor mismatch in CMOS-based chemical imagers. Designed using an $8 \times 8$ array comprising of $\mathrm{pH}$ sensitive Ion Sensitive Field Effect Transistors (ISFETs), the chemical imager is capable of implementing a gradient based calibration algorithm by biasing programmable-gate (PG) ISFETs at a common operating point when exposed to a solution of homogenous pH. The system was fabricated in a typical $0.35 \mu \mathrm{m}$ CMOS technology and demonstrated a fast rate of convergence $(500 \mathrm{~ms}$ per iteration) while a convergence accuracy of $45 \mathrm{mV}$ on a gain of $10(0.5 \%$ relative standard error and $2 \%$ pixel-to-pixel variation $)$ was achieved. A maximum $\mathrm{pH}$ sensitivity of $57 \mathrm{mV} / \mathrm{pH}$ is also reported.
\end{abstract}

Index Terms-lab-on-chip, ISFET, chemical imager, calibration, array

\section{INTRODUCTION}

$\mathbf{T}$ HE analysis of biological systems and miniaturisation of analytical chemical methods are few of the driving forces behind research in the field of lab-on-chip. Lab-onchip is a multi-disciplinary approach to designing integrated micro-scale devices for monitoring and performing biochemical assays. Technology miniaturisation allows for controlled transport and manipulation of biological molecules and cells [1], [2], while spatiotemporal chemical and optical detection can be achieved by integrating CMOS based micro-sensors onto a single platform [3]. CMOS technologies provide an excellent means to meeting modern day performance criteria through very large-scale integrated (VLSI) circuits with complex multiplexing schemes, embedded sensors, programmable memory, batch fabrication and low cost.

Since its invention in the 1970s [4], Ion-Sensitive Field Effect Transistors (ISFETs) have demonstrated great potential for being integrated into micro-chemical sensing systems [5], [6]. The ISFET is essentially a floating gate Metal-oxidesemiconductor FET (MOSFET) where the gate oxide is replaced by a sensing membrane. Depending on the specificity of the membrane, it can be used to detect various analytes such as $\mathrm{H}^{+}, \mathrm{Na}^{+}$and $\mathrm{K}^{+}$. One of the main advantages of ISFETs is their ability to be tesselated to form large chemical sensor arrays, which are used for detecting spatiotemporal

Manuscript received XXX, 2010.

C. Z. D. Goh is with the Department of Bioengineering, Imperial College London, SW7 2AZ, UK. (email: zheng.goh06@imperial.ac.uk)

P. Georgiou, T. G. Constandinou, T. Prodromakis and C. Toumazou are with the Centre for Bio-Inspired Technology (Institute of Biomedical Engineering and Dept. of Electrical and Electronic Engineering), Imperial College London, SW7 2AZ, United Kingdom (tel: +44 207594 0701; fax: +44 2075940704 email: \{pantelis,t.constandinou,t.prodromakis,c.toumazou\}@imperial.ac.uk). ionic concentration profiles. Potential applications include the monitoring of electrochemical activity of a living cell population [7] as well as multi-parameter detection [8].

Despite technological advancements of CMOS-based ISFETs, they often suffer from large threshold voltage mis-match and drift [9]. These non-idealities are largely due to trapped charge which mainly reside in the passivation layer of the floating gate of the device [10]-[12]. So far, few efforts have proved to be effective in minimising non-idealities within an array of ISFETs. In [10], UV radiation was used to match the threshold voltages of a $2 \times 2$ ISFET array. However, this method required an external calibration source and threshold voltages only converged after $10 \mathrm{~h}$ of UV exposure. Electron tunnelling and pFET hot-electron injection can be used to automatically adapt the charge on a floating gate amplifier [13]. This technique eliminates drift by continuously restoring the desired operating point and minimizes device mismatch in an array of floating gate amplifiers. In [14] hot-electron injection was used to change the charge on the floating gate of the ISFET and program it's threshold voltage. This however required programming voltages of $5 \mathrm{~V}$ or greater and was susceptible to degradation of the gate oxide. In [15], a Programmable Gate (PG) ISFET was proposed, whereby the ISFET operating point could be programmed by applying voltage bias to a control gate capacitor attached to the floating gate of the device. This allowed to counteract the effects of trapped charge as well as drift.

In this paper, we present a novel auto-calibration technique based on the PG-ISFET approach. This method was demonstrated via an $8 \times 8$ chemical imager that was fabricated in a commercially available $0.35 \mu \mathrm{m}$ CMOS process. The system overcomes the need for an external UV source and long threshold voltage convergence time. The paper is organised as follows: the system architecture is described in Section II, with the fabrication of the device outlined in Section III. In Section IV, the proposed calibration algorithm is described while supporting measured results are presented in Section V.

\section{SySTEM OVERVIEW}

The top level system architecture of the chemical imager is shown in Fig. 1. It is comprised of: an $8 \times 8$ array of PGISFETs that can capture the chemical image, a DAC that is driven by a lookup table $(8 \times 8 \mathrm{RAM})$ and is programmed with the programmable gate voltages of each pixel, a Programmable Gain Amplifier (PGA) for signal amplification, a 10-bit ADC for data acquisition and the timing circuits used to clock each pixel sequentially. 


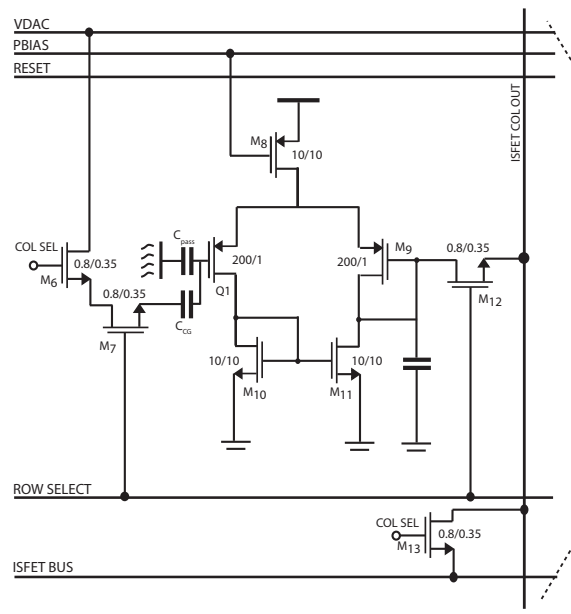

(a)

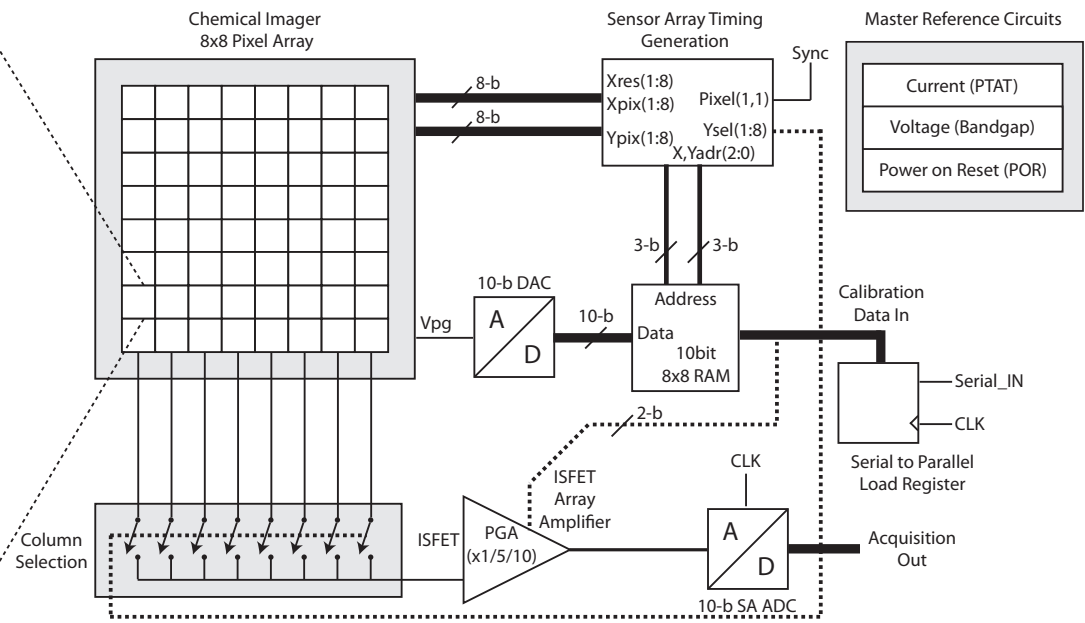

(b)

Fig. 1. System overview: (a) a schematic of the integrated PG-ISFET pixel (left), and a top level architecture of the system (right).

\section{A. PG-ISFET Integrated Pixel}

The schematic of a PG-ISFET pixel is shown in Fig. 1. Each PG-ISFET is biased using a unity-gain buffer interface at pixel-level. The outputs are switched (i.e. rows selected) into a shared column bus at the pixel level while output columns are selected at the column header.

The PG-ISFET floating gate voltage $V_{F G}$ is established by a weighted sum of the voltages applied to the reference electrode $V_{G}^{\prime}$, and the control gate potential $V_{C G}$ :

$$
\begin{gathered}
V_{F G}=\frac{V_{G}^{\prime} \cdot C_{p a s s}+V_{C G} \cdot C_{C G}+V_{S} \cdot C_{G S}+V_{D} \cdot C_{G D}}{C_{T}} \\
C_{T}=\left(C_{o x} / / C_{d}+C_{b}+C_{g s}+C_{g d}+C_{p a s s}+C_{C G}\right)
\end{gathered}
$$

where $C_{o x}, C_{d}, C_{g s}$ and $C_{g d}$ follow the same definition as a conventional MOSFET [16]. When the ISFET is biased, free-ions that exist in the solution bind to the passivation surface causing an accumulation of charge, which in turn modulates the floating gate voltage of the device, thus creating a dependance on $\mathrm{pH}$ according to:

$$
V_{G}^{\prime}=V_{\text {ref }}-V_{t c}-\gamma+\frac{2.3 \alpha k T}{q} p H
$$

whereby $V_{t c}$ represents the effect of non-idealities (i.e. trapped charge and pixel mismatch), $V_{\text {ref }}$ is the bias voltage of the reference electrode, $U_{t}$ is the thermal voltage of the device, $\gamma$ is a grouping of non-chemically related potentials and $\alpha$ is a number ranging from $0-1$ that describes the reduction in sensitivity from the Nernstian response, typically $59 \mathrm{mV} / \mathrm{pH}$ [4]. From Equation 1 we can see that the control gate voltage $V_{C G}$ can be used to counteract the effects of $V_{t c}$ as was demonstrated in [15].

\section{B. System operation}

The system uses a common programming/calibration interface based on the SPI protocol, to load data serially into the RAM [17], [18]. This data is comprised of $8 \times 8 \times 10$-bit words for the chemical imager calibration and an additional 2 bit word to control the programmable amplifier gain. Master reference circuits are also common providing a $1 \mu \mathrm{A}$ PTAT generated current bias, $1.21 \mathrm{~V}$ bandgap voltage reference and power on reset signals to the various circuits.

A timing generator based on a state-machine first defines the different phases and array control signals (eg. $x$ and $y$ pixel select signals) to poll through the sensor pixel array. The outputs are sampled using a single 10-bit successive approximation analogue-to-digital converter. The output signal is then buffered through a Programmable Gain Amplifier (PGA) and passed to the converter for data acquisition. The PGA is designed as a non-inverting operational amplifier with programmable feedback networks setting the gain.

A key innovation of this system is that the programmable gate input is fed from a DAC that is driven by a lookup table (RAM-based loaded at initialization). This has been implemented to calibrate out sensor non-idealities including drift, spatial (static) noise and gain mismatch.

\section{FABRICATED SYSTEM}

The proposed experimental platform was designed and fabricated in a commercially-available $0.35 \mu \mathrm{m}$ CMOS technology. The array is described elsewhere [17], [18] and combines two additional sub-systems (optical detection and magnetic actuation) to the chemical sensing modality. In this paper, only the chemical sensing sub-system is discussed. A microphotograph of the fabricated system is shown in Fig. 2(a), illustrating the chip floorplan. Fig. 2(b) shows the individual components within the pixel footprint. The pixel pitch is 150 $\mu \mathrm{m}$.

The PG-ISFET device is a p-MOS transistor designed with electrical dimensions of $W_{e l} / L_{e l}=200 \mu \mathrm{m} / 1 \mu \mathrm{m}$, offering high transconductance efficiency and chemical sensing dimensions of $W_{\text {chem }} / L_{\text {chem }}=50 \mu \mathrm{m} / 50 \mu \mathrm{m}$. A control gate capacitance $\left(C_{C G}\right)$ of $500 \mathrm{fF}$ was used to offer compensation in the range of $\pm 6 \mathrm{~V}$ using a $3.3 \mathrm{~V}$ supply. 


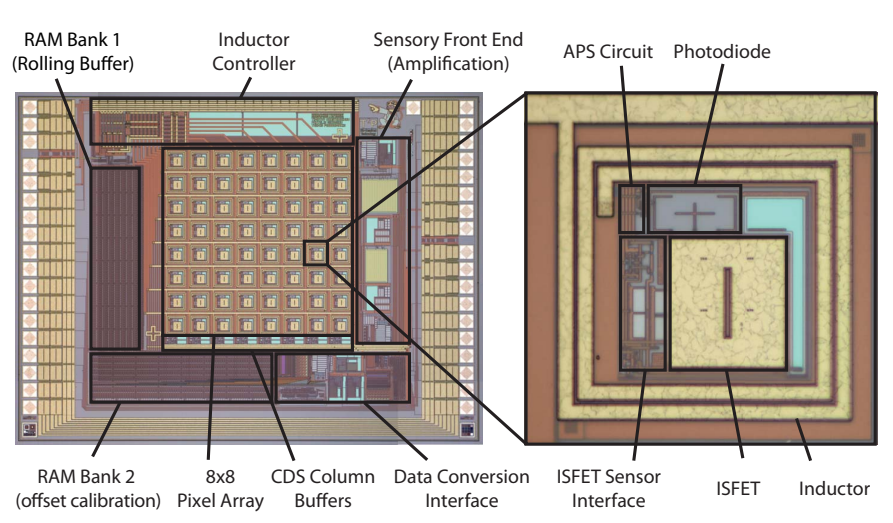

Fig. 2. Microphotograph and overlaid floorplan of: (a) complete system (left) and (b) multimodal pixel (right). A full description of the fabricated system is provided in [17].

\section{A. Instrumentation}

The chemical imager chip and various hardware components were integrated onto a single platform as shown in Fig. 3a. The chemical imager is programmed and controlled from a MATLAB GUI interface, via a Microchip 18LF4680 PIC microcontroller. Communication with the PC was established using a standard UART interface, and SPI pins were set up to program the chip and external DACs.

A finite state machine was implemented to facilitate the transfer of instruction bits between MATLAB and the microcontroller as well as for setting up software handshake routines during real time data acquisition. The sensor array is polled by a clock signal generated by the microcontroller, while each end-of-conversion (EOC) signal triggers the microcontroller to sample a 10-bit digital output word from the chip. The sampled data is then ordered and stored in memory. Completion of data acquisition from a single frame is followed by transferring the data to MATLAB to be displayed as chemical images.

An auxiliary cartridge shown in Fig. $3 b$ was designed for wire bonding and encapsulating the chemical imager. The cartridge also provides an elevated platform for fluidic assays to be conducted on. The chip was encapsulated using globtop resin to provide mechanical support for bond wires and to prevent contamination of the device from chemicals. The cartridge is interfaced to the development board via corresponding connectors. A $2 \mathrm{ml}$ fluidic well was also fabricated and adhered to the cartridge using epoxy resin. An external $\mathrm{Ag} / \mathrm{AgCl}$ electrode was used as a fluid reference which was grounded on the PCB.

\section{Calibration Algorithm}

Using an array of PG-ISFETs offers the capability to calibrate out sensor non-idealities by biasing each PG-ISFET at a common operating point using each pixel's individual control gate voltages stored in the RAM. The calibration process aims to bias the PG-ISFET array at mid supply range (1.65V) when the device is exposed to $\mathrm{pH} 7$ buffer solution, achieving the maximum dynamic range for a $3.3 \mathrm{~V}$ supply.

Calibration is achieved when the mean squared error between the PG-ISFET array output and the operating point is

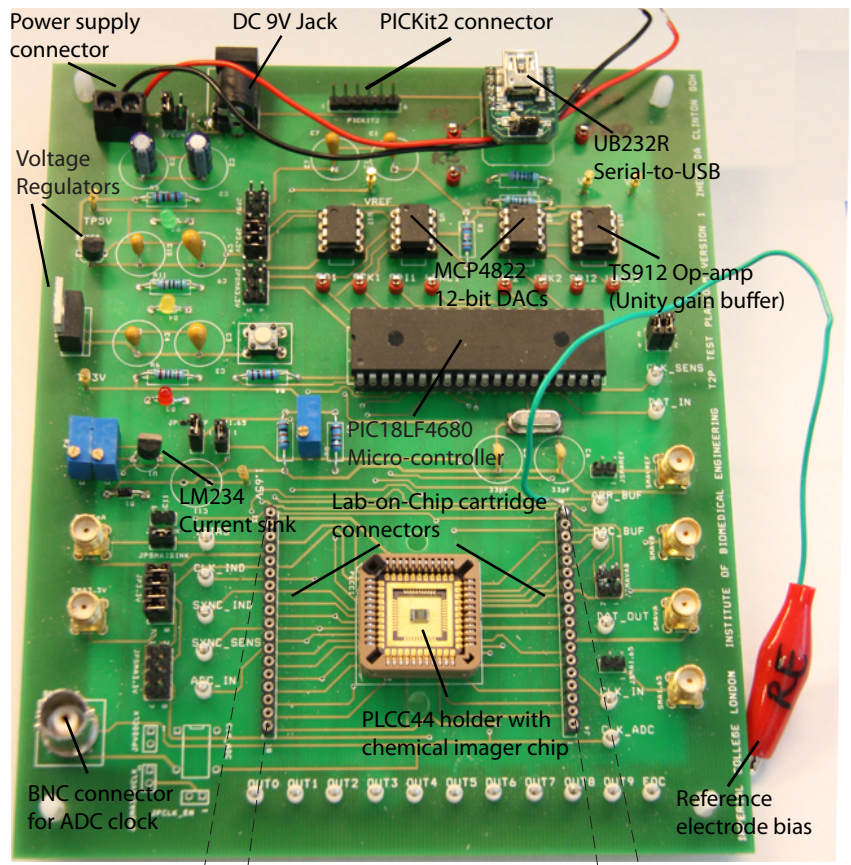

(a)

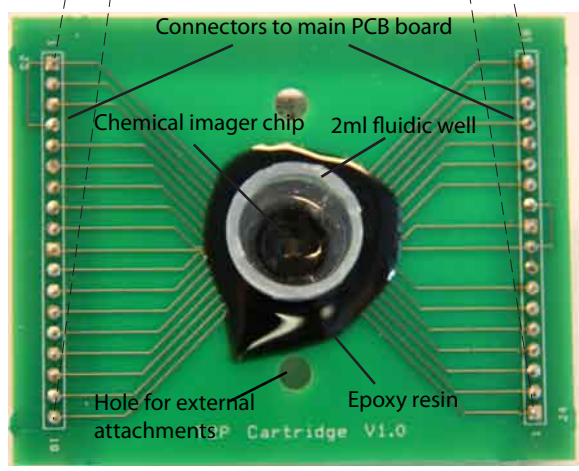

(b)

Fig. 3. Hardware instrumentation: (a) development board consisting of microcontroller and peripheral electronics and (b) chemical imager cartridge.

minimised:

$$
f(x)=\frac{1}{N} \sum_{i=1}^{64}\left(V_{\text {out }}\left(x_{i}\right)-V_{\text {op }}\right)^{2}
$$

where the mean squared error $f(x)$ is a function of the programmable gate voltage $x \in \mathbb{R}^{64}$. $V_{\text {out }}(x)$ is the chemical output and $V_{o p}$ is the operating point of the ISFET array. $f$ is minimised when:

$$
\nabla f(x)=\frac{2}{N} \sum_{i=1}^{64}\left(V_{\text {out }}\left(x_{i}\right)-V_{\text {op }}\right) \nabla V_{\text {out }}(x)=0
$$

A general algorithm which can be used to optimise the problem involves searching for a sequence $\left\{x_{k}\right\}$ that starts from an initial point $x_{0}$, while converging to the set $\Omega=$ $\left\{x \in \mathbb{R}^{64}: \nabla f(x)=0\right\}$, as described below:

1. Fix a point $x_{0} \in \mathbb{R}^{64}$ and set $k=0$.

2. If $x_{k} \in \Omega$, STOP. Else, compute a direction of research $d_{k} \in \mathbb{R}^{64}$.

3. Compute a step $\alpha_{k} \in \mathbb{R}$ along $d_{k}$. 


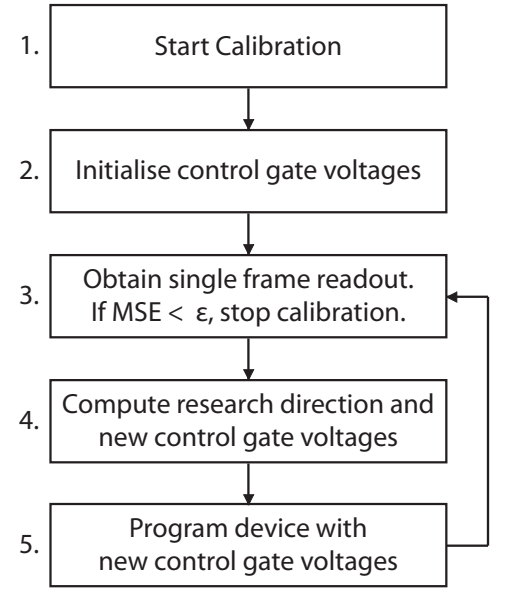

Fig. 4. Calibration algorithm flow chart

4. Let $x_{k+1}=x_{k}+\alpha_{k} d_{k}$. Set $k=k+1$ and go to Step 2.

A gradient based algorithm was implemented for calibrating the chemical imager, where the research direction was selected to be the anti-gradient: $d_{k}=-\nabla f(x)$, and $\alpha_{k}$ can be computed by any line search method such that $f\left(x_{k}+\alpha_{k} d_{k}\right) \leq$ $f\left(x_{k}\right)$. Since $V_{\text {out }}(x)$ is the floating gate voltage of the ISFET, $\nabla V_{\text {out }}(x)$ is the ratio of ISFET capacitances multiplied by the chemical gain, which is a non-zero constant. Therefore, $f$ is minimised only for $V_{\text {out }}\left(x_{i}\right)=V_{\text {op }}$.

The algorithm can be simplified by grouping $\alpha_{k}$ and $\nabla V_{\text {out }}(x)$ as $\rho_{k}<1$, thus eliminating the need for a line search algorithm. Due to noise and quantisation error, searching for a converging sequence may result in oscillations near the operating point. To ensure convergence, the algorithm is modified such that it terminates when $\left\{x_{k}\right\}$ converges to the set $\Phi=\left\{x \in \mathbb{R}^{64}: f(x)<\epsilon\right\}$, where $\epsilon$ is the threshold error. The calibration algorithm is summarised as follows:

1. Fix the initial control gate bias $x_{0}=1.65 \mathrm{~V}$ and set $k=0$.

2. Poll the sensor array and obtain data from a single frame. Compute $f(x)$. If $f(x)<\epsilon$, STOP.

3. Else, set $d_{k}=V_{\text {op }}-V_{\text {out }}\left(x_{i}\right)$.

4. Compute new control voltage $x_{k+1}=x_{k}+d_{k} \rho_{k}$ and set $k=k+1$.

5. Re-program the device with new control gate voltages and go to Step 2.

This algorithm optimises the control gate vector through a negative feedback system based on the difference between the operating point and the actual readout. During each iteration, the sensor array is polled and data from a single frame is acquired. If the terminating condition is not met, the readout data is used to compute new control gate vectors which form a $8 \times 8 \times 10$-bit instruction set. The instruction set is then sent to the microcontroller to be clocked into the RAM block of the chemical imager. As the chemical readout converges to the operating point, the step size reduces and fine tunes the search process. Once the optimum control gate vector is found, the algorithm is terminated. A flowchart of the calibration algorithm is shown in Fig. 4.

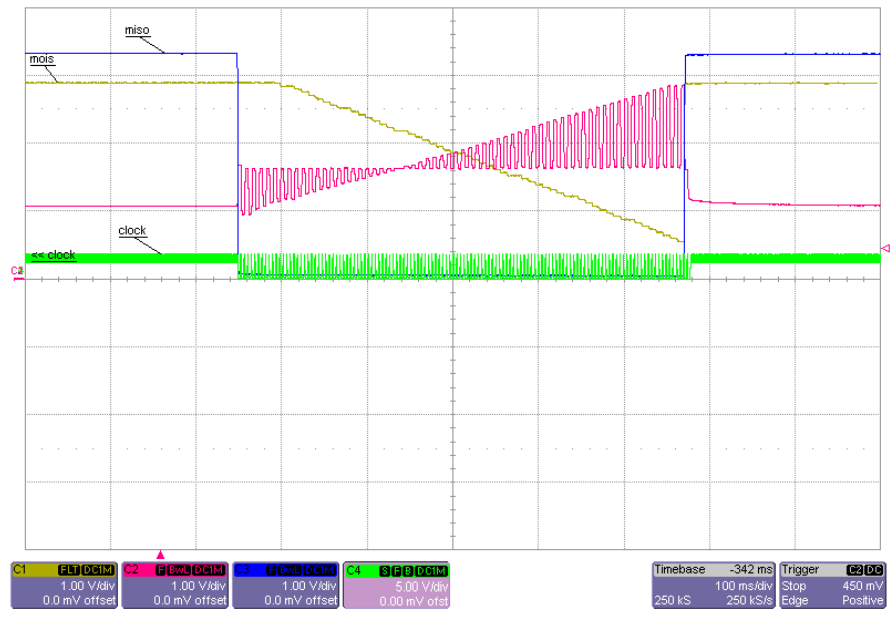

Fig. 5. Polling a frame from the sensor array. The output of each pixel is the red trace, the control gate voltage is the yellow trace, the CTS signal is the blue trace and the EOC signal is the green trace. The monotonically increasing chemical output is inter-leaved with optical readout data [17], [18].

Changing the values of $\rho_{k}$ and $\epsilon$ will affect the speed of convergence and accuracy of the algorithm. For example, choosing a small step size $\rho_{k}$ improves accuracy yet decreases the speed of convergence, while choosing a large threshold error $\epsilon$ leads to a faster rate of convergence at the expense of accuracy. The selection of appropriate values for $\rho_{k}$ and $\epsilon$ optimises the performance of the calibration algorithm and is discussed in Section V.

\section{Measured Results}

\section{A. Data Acquisition and PG-ISFET Biasing}

The functionality of the PG-ISFET array was assessed by programming a series of control gate vectors into the chemical imager. When each pixel is addressed, a 10-bit instruction word is decoded and is used to bias a PG-ISFET. Fig. 5 illustrates the sensor readout from the chemical imager without exposure to fluid. A monotonically decreasing control gate vector was programmed (yellow trace) and the corresponding chemical readout was recorded (red trace). Since the floating gate of the PG-ISFET is interfaced to the inverting input of the PGA and compared against mid-supply, the chemical readout increases with decreasing control gate bias.

\section{B. Sensor Calibration}

The algorithm is dependent on two user defined parameters: step size $\rho_{k}$ along the research direction and the mean squared error threshold $\epsilon$. Since the output is scaled by the chemical gain, the step size was arbitrarily set to $\rho_{k}=\frac{1}{G_{c h e m}}$. The inverse relationship is practical as a change in control gate bias leads to a larger change in output voltage when a larger gain is utilized.

To determine the optimal error threshold $\epsilon, 20$ iterations of the calibration algorithm were used to monitor variations in the mean squared error, as shown in Fig 6. Oscillations due to noise and quantisation error were observed after the tenth iteration. The minimum error also increases with chemical gain 


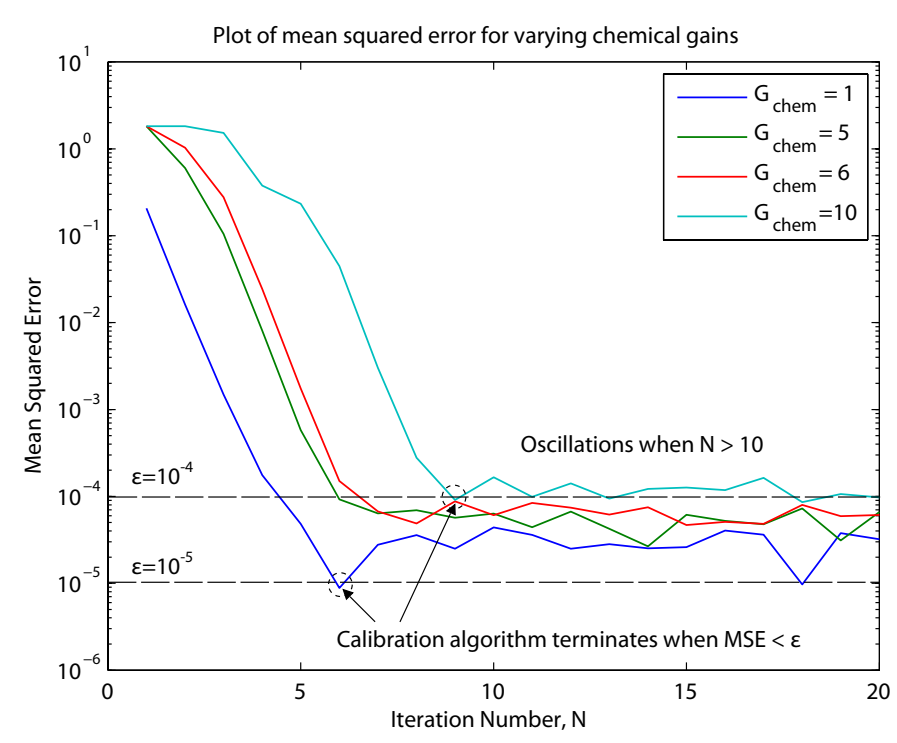

Fig. 6. Plot of the mean squared error against iteration number. Oscillations are observed for $\mathrm{N}>10$, as the algorithm attempts to search for an optimum control gate vector but is unable to do so due to noise and quantisation error.

due to the scaling of 10-bit DAC resolution. A gain of 1 results in a resolution of $3.2 \mathrm{mV}$, and a minimum mean squared error of $1 \times 10^{-5}$, which is 10 times less of that when $G_{c h e m}=10$. Hence $\epsilon$ was chosen to be a function of $G_{\text {chem }}$ where $\epsilon=$ $G_{\text {chem }} \times 10^{-5}$, as illustrated in Fig 6 .

The implemented algorithm was used to bias the chemical imager at $V_{\text {out }}(x)=1.65 \mathrm{~V}$ using a $\mathrm{pH} 7$ buffer solution, where it demonstrated a fast rate of convergence (approximately $500 \mathrm{~ms}$ for each iteration). The auto-calibration process is illustrated in Fig. 8, whereby the control gate vector is modified with each iteration, such that convergence is achieved when all PG-ISFETs are biased at a common operating point. What can be seen is the output of the chemical imager is initially around $2.5 \mathrm{~V}$ and converges to an operating point of $1.65 \mathrm{~V}$ across all the pixels once the calibration is completed in seven iterations. The algorithm achieved a convergence accuracy of $45 \mathrm{mV}$ ( $2 \%$ pixel-to-pixel variation) on a gain of 10 and an average standard deviation of $9 \mathrm{mV}(0.5 \%$ relative standard error).

\section{C. $p H$ Sensitivity}

The $\mathrm{pH}$ sensitivity of the platform was evaluated with solutions of varying $\mathrm{pH}$. The device was first calibrated with a pH 7 buffer solution and the chemical readout was recorded with solutions of known $\mathrm{pH}$. In all experiments, an $\mathrm{Ag} / \mathrm{AgCl}$ reference electrode was used to maintain the fluid potential at $0 \mathrm{~V}$ while the chemical readout was allowed to settle for 120 s before a measurement was taken. Fig 7 demonstrates the system response to varying $\mathrm{pH}$ levels. The chemical readout from all 64 pixels was averaged for each $\mathrm{pH}$ level and fitted to a first order polynomial using the method of least squares. The system demonstrated a chemical sensitivity of $57 \mathrm{mV} / \mathrm{pH}$ on a gain of 10 , which is very close to the Nernstian sensitivity of $59 \mathrm{mV} / \mathrm{pH}[4]$.

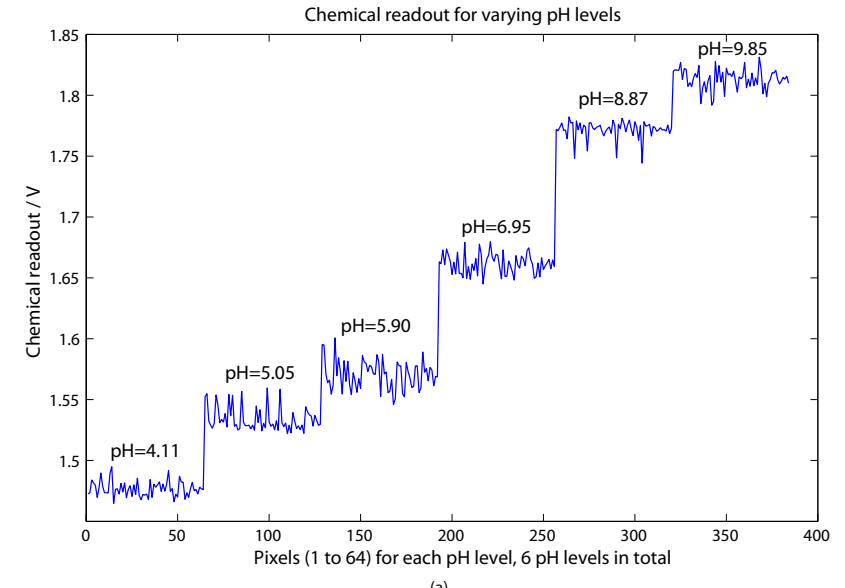

(a)

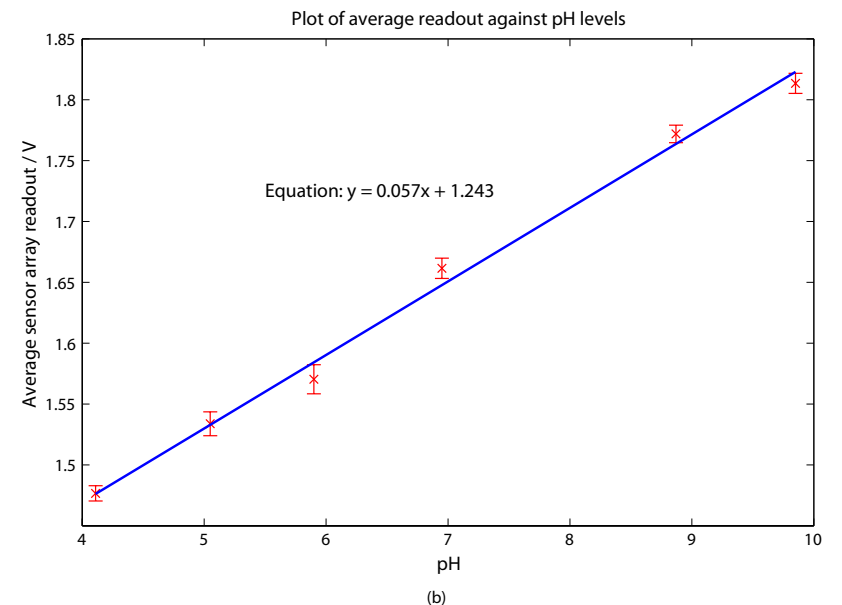

Fig. 7. Chemical readouts for varying $\mathrm{pH}$ levels. (a) Recordings from all pixels for each $\mathrm{pH}$ level, with a total of $6 \mathrm{pH}$ levels. (b) Averaged array readout for varying $\mathrm{pH}$ levels. Data is shown as the mean \pm standard deviation

\section{CONCLUSiON}

A novel auto-calibration technique for eliminating sensor non-idealities has been presented in this paper. We described a novel $8 \times 8$ chemical imaging array that enables the calibration of individual programmable gate-ISFET pixels. The system specifications are summarised in Table I. One key advantage of this system is that all circuitry is integrated onto a single platform and there is no requirement for any external calibration source. An auto-calibration algorithm was also implemented to optimise the control gate vector required to bias the PGISFET array at mid-supply voltage $(1.65 \mathrm{~V})$. This is carried out by iteratively updating the control gate vector based on the error between the desired operating point and actual readout. Calibration data stored in a lookup table is then used to bias the PG-ISFET array at the desired operating point. To the best of our knowledge this is the first in-pixel ISFET calibration technique (see Table II for a comparison with other work).

The algorithm was used to calibrate the chemical imager using a $\mathrm{pH} 7$ buffer solution, where it demonstrated a fast rate of convergence (approximately 500ms for each iteration) and a convergence accuracy of $4.5 \mathrm{mV} \times G_{\text {chem }}$ (pixel-to-pixel variation), where $G_{c h e m}$ is the gain of the PGA. Auto-calibration was also employed in characterising the $\mathrm{pH}$ sensitivity of the chemical imager. The system demonstrated a sensitivity of 

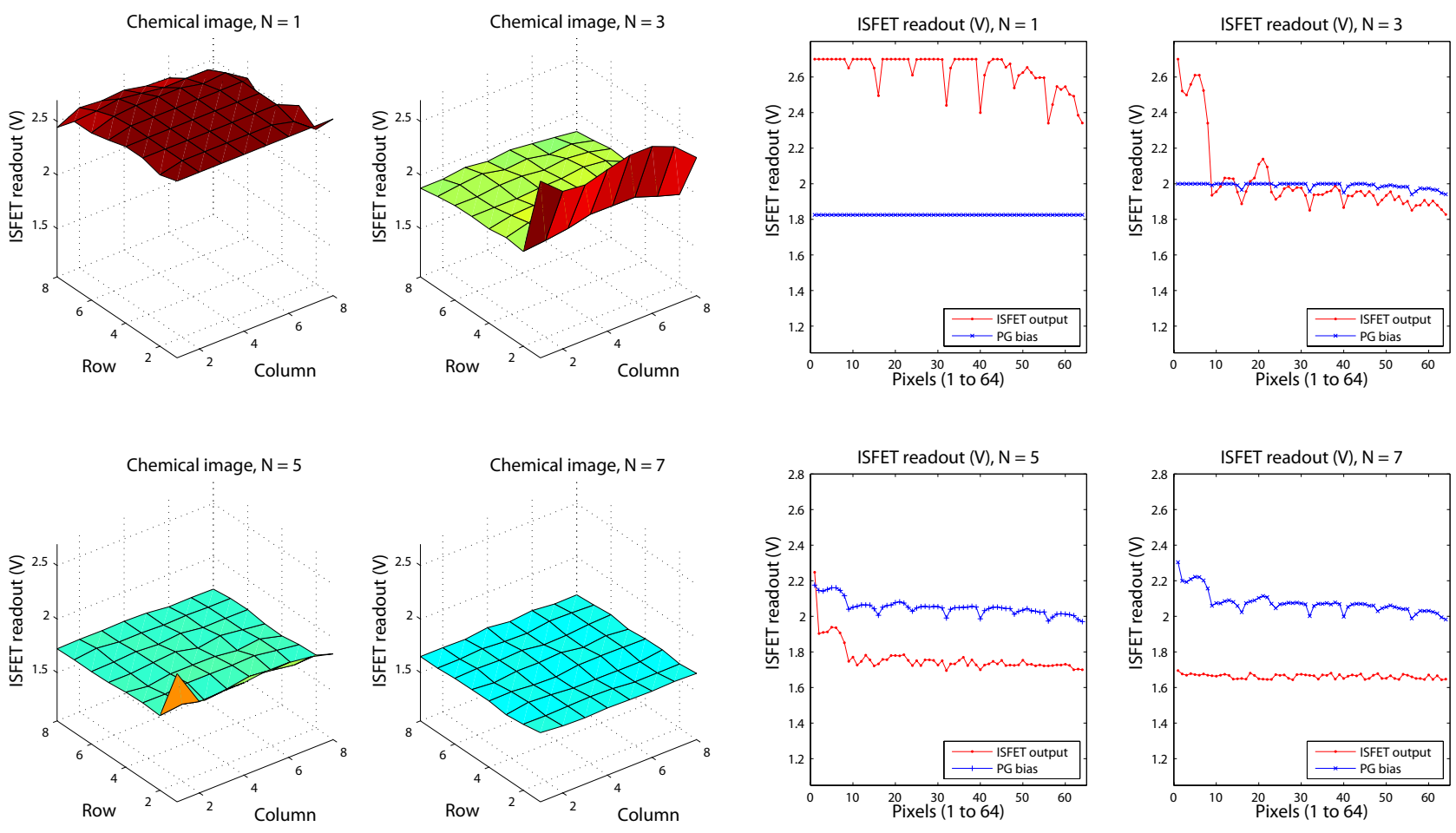

(b)

Fig. 8. The calibration process (even numbered iterations are omitted): (a) acquired chemical images from each iteration of the PG-ISFET array calibration process (left). (b) the chemical readout (red trace) converges to a common operating point when the control gate vector (blue trace) is optimised.

TABLE I

SYSTEM PROPERTIES AND PERFORMANCE SUMMARY

\begin{tabular}{ll}
\hline Technology, Supply voltage & AMS $0.35 \mu \mathrm{m}$ 2P4M CMOS, 3.3V \\
\hline Sensing area, fill factor & $2500 \mu \mathrm{m}, 11.1 \%$ \\
Sense resolution, range & $0.01 \mathrm{pH}, 2-12 \mathrm{pH}$ \\
Frame rate & $<25 \mathrm{FPS}$ \\
Vt-calibration range & $\pm 6 \mathrm{~V}(10$ bit tuning $)$ \\
\hline Accuracy & $\mu=1.65 \mathrm{~V}, \sigma=9 \mathrm{mV}$ \\
pH sensitivity & $57 \mathrm{mV} / \mathrm{pH}$ \\
Rate of convergence & $500 \mathrm{~ms} \mathrm{per} \mathrm{iteration}$ \\
\hline Die dimensions & $2895 \mu \mathrm{m} \times 2155 \mu \mathrm{m}$ \\
System device count & 57,784 \\
Array size & $8 \times 8$ pixels \\
ADC res., conversion time & $10-$ bit, $10 \mu \mathrm{s}$ \\
\hline
\end{tabular}

$57 \mathrm{mV} / \mathrm{pH}$ and could be used to image variations in solution $\mathrm{pH}$. The system out-performs previous efforts at minimising sensor non-idealities in terms of speed of convergence and allows all PG-ISFETs in the array to respond to a change in solution $\mathrm{pH}$ with the same relative change in output voltage. The high speed of convergence also allows the auto-calibration technique to remain practical if the array was scaled up in size.

The PG-ISFET utilised a control gate capacitor $C_{C G}$ which increases the total capacitance $C_{T}$ on the floating gate node. This effectively decreases voltage contribution due to a $\mathrm{pH}$ change by dividing down the voltage generated on the sensing membrane $V_{G^{\prime}}$, but is offset by increasing $G_{c h e m}$. However, a higher gain amplifies the calibration error, which results in a reduction in convergence accuracy. To improve the system's convergence accuracy, a higher resolution DAC and noise filters can be implemented in future versions. By reducing quantisation error and noise, we aspire to improve the pixelto-pixel variation while maintaining $\mathrm{pH}$ sensitivity.

The proposed auto-calibration mechanism also creates opportunities for designing a calibration routine to auto-correct chemical drift. By continuously updating the control gate vector, the desired operating point can be maintained to improve the readout stability and reliability of the platform. We hope that in future versions, the successful elimination of chemical drift and an improvement in convergence accuracy will advance CMOS-based chemical imagers towards becoming commercially viable systems.

\section{REFERENCES}

[1] H. Andersson and A. van den Berg, "Microfluidic devices for cellomics: a review," Sensors and Actuators B: Chemical, vol. 92, no. 3, pp. 315 $-325,2003$

[2] M. Yang, C.-W. Li, and J. Yang, "Cell docking and on-chip monitoring of cellular reactions with a controlled concentration gradient on a microfluidic device," Analytical Chemistry, vol. 74, pp. 3991-4001, Aug. 2002.

[3] T. C. W. Yeow, M. R. Haskard, D. E. Mulcahy, H. I. Seo, and D. H. Kwon, "A very large integrated ph-isfet sensor array chip compatible with standard cmos processes," Sensors and Actuators B: Chemical, vol. 44, no. 1-3, pp. $434-440,1997$.

[4] P. Bergveld, "Thirty years of isfetology - what happened in the past 30 years and what may happen in the next 30 years," Sensors and Actuators B: Chemical, vol. 88, no. 1, pp. 1-20, 2003.

[5] J. Bausells, J. Carrabina, A. Errachid, and A. Merlos, "Ion-sensitive field-effect transistors fabricated in a commercial CMOS technology," Sensors and Actuators B: Chemical, vol. 57, no. 1-3, pp. 56-62, 1999. 
TABLE II

COMPARISON WITH OTHER WORK

\begin{tabular}{|c|c|c|c|}
\hline $\begin{array}{c}\text { Year } \\
\text { Reference }\end{array}$ & $\begin{array}{r}2008 \\
{[10]}\end{array}$ & $\begin{array}{r}2010 \\
{[19]}\end{array}$ & $\begin{array}{c}2011 \\
\text { This work }\end{array}$ \\
\hline Technology & $\begin{array}{l}\text { AMS } 0.35 \mu m \\
\text { CMOS } 2 \mathrm{P} 3 \mathrm{M}\end{array}$ & $0.35 \mu \mathrm{m}$ CMOS & $\begin{array}{l}\text { AMS } 0.35 \mu m \\
\text { CMOS } 2 \mathrm{P} 4 \mathrm{M}\end{array}$ \\
\hline $\begin{array}{l}\text { Die size } \\
\left(m m^{2}\right)\end{array}$ & 9.4 & 25.85 & 6.38 \\
\hline $\begin{array}{l}\text { Array } \\
\text { size }\end{array}$ & $2 \times 2$ array & $40 \times 1$ array & $8 \times 8$ array \\
\hline $\begin{array}{l}\text { ISFET } \\
\text { size } \\
\left(\mu m^{2}\right)\end{array}$ & 164 & $\mathrm{n} / \mathrm{a}$ & 2500 \\
\hline Method & $\begin{array}{l}\text { UV radiation \& } \\
\text { bulk-substrate } \\
\text { biasing }\end{array}$ & $\begin{array}{c}\text { Digital } \\
\text { calibration with } \\
\text { summing } \\
\text { amplifiers }{ }^{1}\end{array}$ & $\begin{array}{l}\text { Gradient-based } \\
\text { optimisation of } \\
\text { PG-ISFET } \\
\text { tuning }\end{array}$ \\
\hline Calibration & Off-chip & System-level & In-pixel \\
\hline$\underset{\text { sensitivity }}{\mathrm{pH}}$ & $46 \mathrm{mV} / \mathrm{pH}$ & $\mathrm{n} / \mathrm{a}$ & $57 \mathrm{mV} / \mathrm{pH}^{2}$ \\
\hline $\begin{array}{l}\text { Convergence } \\
\text { rate }\end{array}$ & $10 \mathrm{~h}$ & $\mathrm{n} / \mathrm{a}$ & $\begin{array}{l}500 \mathrm{~ms} \text { per } \\
\text { iteration }(\approx 7 \text { to } \\
10 \text { iterations })\end{array}$ \\
\hline Accuracy $\ddagger$ & $10 \mathrm{mV}$ & $3 m V^{3}$ & $4.5 \mathrm{mV} \times G^{4}$ \\
\hline
\end{tabular}

${ }^{\dagger}$ After calibration. ${ }^{\ddagger}$ Pixel-to-pixel variation

${ }^{1}$ 10-bit DAC biasing. ${ }^{2}$ Gain of $10 .{ }^{3}$ Gain of $1 .{ }^{4}$ PGA gain .

[6] L. Shepherd and C. Toumazou, "Weak inversion isfets for ultra-low power biochemical sensing and real-time analysis," Sensors and Actuators B: Chemical, vol. 107, no. 1, pp. 468 - 473, 2005.

[7] L. Lorenzelli, B. Margesin, S. Martinoia, M. T. Tedesco, and M. Valle, "Bioelectrochemical signal monitoring of in-vitro cultured cells by means of an automated microsystem based on solid state sensor-array," Biosensors and Bioelectronics, vol. 18, no. 5-6, pp. 621 - 626, 2003.

[8] A. Poghossian and M. Schning, "Detecting both physical and (bio-) chemical parameters by means of isfet devices," Electroanalysis, vol. 16, no. 22, pp. 1863-1872, 2004.

[9] P. A. Hammond, D. Ali, and D. R. S. Cumming, "Design of a single-chip ph sensor using a conventional 0.6- $\mu \mathrm{m}$ CMOS process," IEEE Sensors Journal, vol. 4, no. 6, pp. 706-712, 2004.

[10] M. Milgrew and D. Cumming, "Matching the Transconductance Characteristics of CMOS ISFET Arrays by Removing Trapped Charge," IEEE Trans. Electron Devices, vol. 55, no. 4, pp. 1074-1079, 2008.

[11] T. Prodromakis, P. Georgiou, K. Michelakis, and C. Toumazou, "Effect of mobile ionic-charge on cmos based ion-sensitive field-effect transistors (isfets)," Proc. IEEE Int. Symp. Circuits and Systems, pp. 2165 -2168, 24-27 2009.

[12] P. Georgiou and C. Toumazou, "Isfet characteristics in cmos and their application to weak inversion operation," Sensors and Actuators B: Chemical, 2009.

[13] D. W. Graham, E. Farquhar, B. Degnan, C. Gordon, and P. Hasler, "Indirect programming of floating-gate transistors," IEEE. Trans. Circuits and Systems I, vol. 54, pp. 951 -963, may. 2007.

[14] P. Georgiou and C. Toumazou, "ISFET threshold voltage programming in CMOS using hot-electron injection," IET Electronics letters, vol. 45, p. 1112, 2009.

[15] P. Georgiou and C. Toumazou, "An adaptive cmos-based pg-isfet for ph sensing," Proc. IEEE Int. Symp. Circuits and Systems, pp. 557-560, May 2009.

[16] E. Vittoz and J. Fellrath, "CMOS analog integrated circuits based on weak inversion operations," IEEE Journal of Solid-State Circuits, vol. 12 , no. 3, pp. 224-231, 1977.

[17] T. G. Constandinou, P. Georgiou, T. Prodromakis, and C. Toumazou, "A cmos-based lab-on-chip array for the combined magnetic stimulation and opto-chemical sensing of neural tissue," Proc. IEEE Conf. on Cellular Nanoscale Networks and Applications, 2010.

[18] Z. D. C. Goh, P. Georgiou, T. G. Constandinou, T. Prodromakis, and
C. Toumazou, "A cmos-based lab-on-chip array for combined magnetic manipulation and opto-chemical sensing," Proc. IEEE Int. Symp. Circuits and Systems, 2011.

[19] D. M. Garner, H. Bai, P. Georgiou, T. G. Constandinou, S. Reed, L. M. Shepherd, W. Wong, K. T. Lim, and C. Toumazou, "A multichannel dna soc for rapid point-of-care gene detection," Proc. IEEE Solid-State Circuits Conf., pp. 492-493, 2010.

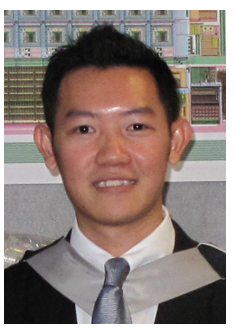

Clinton Z. D. Goh received the M.Eng. degree in Biomedical Engineering in 2010 from Imperial College London. He is currently a research and development engineer with Applied Mesh Pte Ltd, a technological startup based in Singapore. His research interests include circuits and systems for biomedical applications. He received the Bioengineering Student Centenary Prize from Imperial College London in 2010.

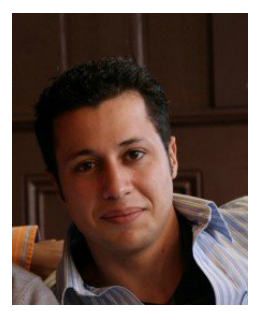

Pantelis Georgiou (AM'05-M'08) received the M.Eng. degree in Electrical and Electronic Engineering in 2004 and the Ph.D. degree in 2008 both from Imperial College London. He then moved to the Institute of Biomedical Engineering (also at Imperial) where he was appointed as a Research Fellow until joining academic faculty in 2011. He is currently a lecturer within the Department of Electrical \& Electronic Engineering and is also the head of the Bio-inspired Metabolic Technology Laboratory in the Centre for Bio-Inspired Technology and part of the Medical Engineering Solutions in Osteoarthritis Centre of Excellence. His research includes bio-inspired circuits and systems, CMOS based lab-on-chip technologies and application of micro-electronic technology to create novel medical devices. He conducted pioneering work on the silicon beta cell and is now leading the project forward to the development of the first bio-inspired artificial pancreas for Type I diabetes. Dr Georgiou is a member of the IEEE and IET. He has been elected a member of the BioCAS Technical Committee of the IEEE Circuits and Systems Society.

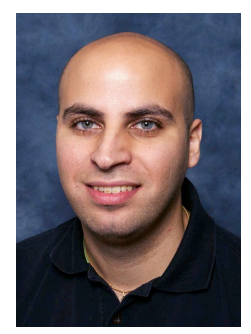

Timothy G. Constandinou (AM'98-M'01-SM'10) received the B.Eng. degree in Electrical and Electronic Engineering in 2001 and the Ph.D. degree in 2005 both from Imperial College London. $\mathrm{He}$ then moved to the Institute of Biomedical Engineering (also at Imperial) where he was appointed to Research Officer in Bionics until joining academic faculty in 2010. He is currently a lecturer within the Department of Electrical \& Electronic Engineering at Imperial College London and is also the deputy director of the Centre for Bio-Inspired Technology. His Research is in the novel application of microtechnology to develop advanced medical devices (implantable, wearable and lab-on-chip) and biologicallyinspired circuits, devices and systems. Dr Constandinou is an IEEE Senior Member, an IET Member and a registered Chartered Engineer. He has been elected a member of the Sensory Systems and BioCAS Technical Committees of the IEEE Circuits \& Systems Society, and also serves on the IET awards committee. 


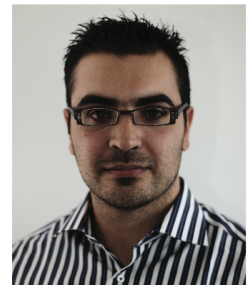

Themistoklis Prodromakis (AM'04-M'08) holds a Corrigan research fellowship in nanoscale science and technology, funded by LSI Logic Inc. and the Corrigan-Walla Foundation, within the Centre for Bio-inspired Technology at Imperial College London. He received his $\mathrm{PhD}$ from the Departmen of Electrical and Electronic Engineering at Imperial College in 2008, during which he successfully pioneered the use of interfacial polarisations for demonstrating miniature passive devices. During his research career he has contributed in several projects in the areas of RF and Microwave Design and particularly Electron Devices, including: miniaturisation techniques MEMS-based phase-shifting topologies, slow-wave filters on laminar architectures, high-k dielectrics and processing techniques for engineering polarisation mechanisms. In 2006 he contributed in setting up the Cleanroom facilities and the Microelectronics Laboratory at the IBE. He recently applied his expertise in the biomedical arena with some examples involving: the development of integrated CMOS chemical sensors, encapsulation techniques and materials and biologically inspired systems.

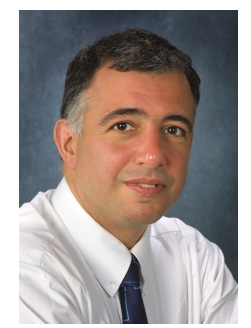

Christofer Toumazou (M'87-SM'99-F'01) is a Professor of Circuit Design, Founder and Executive Director of the Institute of Biomedical Engineering at Imperial College London, UK. Professor Toumazou has made outstanding contributions to the fields of low power analogue circuit design and current mode circuits and systems for biomedical and wireless applications. Through his extensive record of research, he has invented innovative electronic devices ranging from dual mode cellular phones to ultra-low power devices for both medical diagnosis and therapy. He has published over 320 research papers in the field of RF and low power electronics and is a member of many professional committees.. He holds 23 patents in the field, many of which are now fully granted PCT. He is the founder of four technology based companies with applications spanning ultra low-power mobile technology and wireless vital sign monitors (Toumaz Technology Ltd, UK), biomedical devices (Applied Bionics PTE, Singapore), digital audio broadcasting (FutureWaves Pte Taiwan) and DNA detection (DNA Electronics Ltd, UK). These companies employ over $50 \mathrm{RF} /$ low power engineers worldwide many of whom are Professor Toumazou's ex-graduate students. Professor Toumazou was invited to deliver the 2003 Royal Society Clifford Patterson Prize Lecture, entitled "The Bionic Man", for which he was awarded The Royal Society Clifford Patterson bronze medal. He was recently awarded the IEEE CAS Society Education Award for pioneering contributions to telecommunications and biomedical circuits and systems, and the Silver Medal from the Royal Academy of Engineering for his outstanding personal contributions to British engineering. In 2008, he was elected to the grade of Fellow of both the Royal Society and Royal Academy of Engineering. 